\title{
Procedural Generation of Topologic Road Networks for Driving Simulation
}

\author{
Carlos Campos, João Miguel Leitão, João Paulo \\ Pereira, and António Ribas \\ School of Engineering of Porto Polytechnic \\ INESC-TEC \\ Porto, Portugal
}

\begin{abstract}
Virtual environments for driving simulation aimed to scientific purposes require three-dimensional models of realistic road networks. The generation of these networks according to the requirements, if done manually by road design specialists, results in a time consuming task. Procedural generation of road networks comes as a solution to this problem with the creation of complete road networks definition adequate to simulation. This paper proposes a method to automatically generate an optimized definition of very large roads network, in an integrated process, from the selection of nodes in a terrain area, to the network topological definition. The human supervisor can interact with this generation process at any stage, in order to obtain custom road networks definitions. The proposed method reduces the use of specialists for preparing large road networks definitions. These definitions are suitable to integrate into a broader process to create road environments, with different road types, appropriate to conducting scientific experiments in driving simulation.
\end{abstract}

Keywords - Procedural Generation; Road Network; Road Network Design; Driving Simulation.

\section{INTRODUCTION}

Procedural generation of road environments is not only used for entertainment applications but also for scientific applications, that require the use of a driving simulator to conducting scientific work.

The driving simulation for scientific purposes requires the creation of complex and extensive roads networks that represent the region or country which we intended to model. Also, a semantic description of the entire roads network, that allows the parameterization of actors during the simulation and the production of simulation reports, is required.

The road networks created for driving simulation must obey to detailed standards for specification in order to achieve the level of realism adequate for each experimental work. Models of road environments that meet the high standards of realism required by the experimental work in driving simulators can be produced by involving design specialists. This production includes several areas of engineering besides roads engineering and requires the analysis of the road network as a whole. The creation of network models is a task that can become labor intensive and expensive if an automatic method isn't applied. The automatic generation of realistic road networks is therefore very useful [1] [2].

\author{
António Fernando Coelho \\ Engineering Faculty of Porto University \\ INESC-TEC \\ Porto, Portugal
}

In this paper we present the implementation of a prototype for automatic generation of the definition of a road network, to allow the preparation of road environments suitable for driving simulation. A topological road network may be defined by a set of different nodes type (city, town and village) and connections (highway, secondary and rural).

The road network design begins with a definition of nodes that will be interconnected. The method proposed creates a nodes definition on an empty terrain. Beyond the localization, the nodes have attached an importance factor that allows to classify it by typology: cities, towns or villages. Then, the definition of highways, secondary roads and rural road networks are created. From this process, results the topological definition of a network that defines the connections between nodes and the typology of the roads, highways, secondary roads and rural.

In order to produce fully controllable environments, the preparer of the model can interact at any stage of the road network procedural generation process, editing the result produced by any module (definition of nodes and network). This feature is crucial in the generation of virtual environments for driving simulation for scientific purposes.

After obtaining the definition of the road network, it is still necessary to spend resources to produce the road paths, the three-dimensional models of each road and the terrain model. The design project of a road path can become a time consuming task. A way to optimize the process of generating the layout of the road from the topological definition is to use tools for automatic generation to facilitate this task, as presented in [3]. For the generation of de 3D models, procedural modeling techniques can be used, as presented in [4].

In this paper, section 2 presents the state of art of the design of road networks and the relevant related work. The proposed method is presented in section 3 , and in section 4 we present the interactive prototype for the generation of road networks. The results obtained with the implemented prototype are presented in section 5. Section 6 presents the conclusions and propose some guidelines for future work.

\section{RELATED WORK}

\section{A. Roads Network Design}

A network as e.g. a road network may be defined by a set of nodes and connections (1). 


$$
\mathrm{R}=(\mathrm{N}, \mathrm{L})
$$

where $\mathrm{R}$ is the network, $\mathrm{N}$ the set of network nodes and $\mathrm{L}$ is the set of connections between two network nodes.

Each connection associates two distinct nodes (2).

$$
\mathrm{L}=\left(\mathrm{N}_{\mathrm{i}}, \mathrm{N}_{\mathrm{j}}\right)
$$

where $\mathrm{L}$ is the link, $\mathrm{Ni}$ and $\mathrm{N}_{\mathrm{j}}$ are the end nodes of the connection.

A road network can be structured hierarchically in 3 levels: the highway network, the secondary road network and rural road network [2] [5].

Roads are planned to connect two or more points of interest, allowing the movement of people between them, trying to minimize construction costs and operating costs. Construction costs are related to the real world implementation, which also includes costs from earthworks, embankments, expropriations, and others. Operating costs are related to population traveling between the two connected nodes and includes factors, such as: fuel, traveling distance and duration of the trip. On a good road network, passengers should be able to get from their origin to their destination with minimal operation cost and traffic. Planning a road network aims to optimize the costs of construction and operation and also reducing the impact of this network into the environment, trying to fulfill the security levels, meet the service levels of traffic and meet the users' needs.

The design of a road starts with a strategic decision to connect two points on the existing road network. Essentially, this decision is made taking into account parameters such as population growth, level of service, traffic studies and estimated construction cost.

\section{B. Virtual Roads Network}

Several works have been developed for creating road city environments, focusing mainly on urban layout [2] [6] [7] [8] [9]. Typically the networks of urban roads follow a certain pattern: orthogonal, radial or branching [10]. Geuter et al. and Weber et al., presents methodologies to continuous generation of pseudo-infinite city models [5] [11]. A system called Citygen, presented by Kelly and McCabe, aims to create the urban geometry typical of a modern city and is based on a variety of road network patterns, that the user can modify [9]. The final stage of the generation is the construction of buildings.

The results obtained by these methods are usable for most visual simulation applications. The problem with these approaches is the need for an excessive control by the user to obtain realistic models, besides, these methods don't produce extra-urban roads network. Driving simulation experiments typically require mixed road networks, combining roads in urban and rural environments, with road models of good quality and realistic road paths.

Procedural modelling languages, such as L-systems and CGA shapes, are not adequate to produce extensive road paths for driving simulation [6] [8] [12]. It is known that the driver can easily learn the road layout in an experimental work, therefore the repetition of road segments is not advisable. The unknown component of the road path is a very important variable in a driver behavior research [13].

Soon Theo, describes a process based on Autopolis to the automatic creation of realistic cities, where the regions of the cities are located, based on geographical features and cityplanning rules [14]. For example, it is preferable that airports are located on flat land and near coastal areas, to allow future expansion [2]. The process first fixes the important regions of a city, such as the commercial center, historical center, and residential areas. Then, a network of freeways and main roads is built. Finally, a network of streets is built throughout the city.

Sun et al. present a methodology to create a virtual road network based in patterns [10]. They build road patterns based on image-derived templates and rule-based systems. They accept an input map of the population density in each area, and then intelligently produce streets of different patterns based on geographical and urban restrictions, such as elevation and congestion. An approach to follow is the creation of a road network based on the population of a city.

Chen et al. present a methodology to create a road network based in tensor fields [7]. The tensors fields are interactively created and edited to design the road network. This is done in three stages. In the first stage, the user designs some base tensor fields with a drawing interface. Next, the street graph is created by using the hyper streamlines of this tensor field. Finally, the graph is used to create a full three-dimensional model of the city. This method is able to create both grid and radial patterns, and can also generate roads following natural barriers. The user interaction needed is not suitable to the automatically generation of extensive roads networks.

A methodology for generating a road network that integrates different typologies of roads (highways, secondary roads and rural roads) is presented by Galin et al. in [15]. The generation of the global transport network is initiated by creation of the highway network, followed by the secondary network and finally the rural network. Each type of road is created independently. In the end, all the roads are overlapped into a single road network. The way this problem is handled, may require that some segments of the road paths need to be recalculated.

Thomas and Donikian showed a model implementation of a virtual city where several actors (like pedestrians, vehicles and public transport vehicles driven by other drivers circulating in different directions) are included and controlled over the complex urban road environment [16]. The urban environment was created using the interactive modeler VUEMS and based on a terrain definition in a digital elevation map [17]. The use of an interactive modeler is not suitable for the automatic and efficient generation of extensive road environments.

Creating models of realistic roads for driving simulation is usually performed by manual, laborious and slow processes, even when the available interactive tools are applied. Whenever a realistic road environment for driving simulation experiments is needed, a high level of interaction with the preparer is usually necessary, which requires experts in road design. 
An alternative to this problem is to develop a methodology for automatic generation of wide roads networks and extensive road paths, from the definition of nodes, topological definition of the network, to the geometric model generation. Also a semantic description of the road environment should be procedural generated.

\section{PROCEDURAL GENERATION OF TOPOLOGIC ROAD NETWORK}

The proposed method creates, in an integrated process, a complete road environment from the generation of inter-change nodes to the construction of the three-dimensional model [1]. The process of generating the three dimensional model is organized hierarchically in layers, which also gives a semantic description of the road environment, as illustrated in Fig. 1.

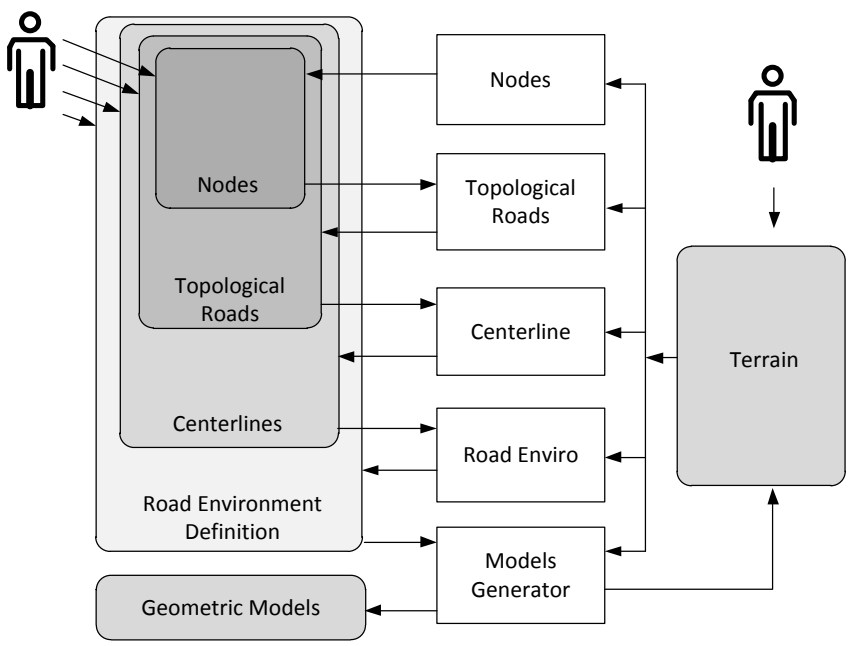

Figure 1. Overview of process workflow.

In this paper, we focus, in the integrated process, from the node definition to the generation of topological road network definition.

In this approach, the procedural generation of a road environment starts at the Nodes module, which creates a definition of interconnection nodes on a given area of the terrain. This module has as input parameters the definition of the area of the terrain where the nodes are distributed and the number of nodes that will be generated. The terrain model can be associated with a definition of constraints that should be considered in the generation of nodes. The Nodes module produces a list of nodes, where each node has associated its location and an importance factor. The importance factor is used to organize the nodes by typology.

The Topological Roads module is aimed at generating the definition of a topological road network. The topological road network is created hierarchically by levels, similar to what happens in other works [2] [5] [15]. The Topological Roads module receive as input the definition of nodes generated by the Nodes module and, depending on the importance factor, organizes the nodes by typologies: cities, towns and villages. Based on this organization, the topological definition of each network is created. The highway network is created from the nodes of the city type, the secondary network is originated from the nodes of the town type, and the rural network is creat- ed from the village nodes. Thus, highways connect cities, secondary roads connect cities and towns, and rural roads connect towns and villages. Additional nodes of inter-connection may be created, with an importance factor of zero.

In the hierarchical process of generating a road network, the preparer of the model can interact at any stage of the process, modifying the result produced by any module and creating a custom layout. For example, the preparer can add new nodes, edit or delete a connection, or edit the definition of the terrain.

This freedom of interaction with the network at any stage of the generation process of the road network is a special contribution of this method that enables producing customized definitions of networks, allowing to fulfill any particular specifications required to the experimental work.

\section{A. Terrain and Nodes}

The generation of nodes can be divided into two subproblems: the generation of terrain definition and the generation of nodes. The land itself must be generated, along with water features and vegetation. Land is typically created by generating a height map that contains an elevation value for each cell. This generation is usually done with some form of fractal noise as a base, that in a simple and fast way define the shape of the terrain [18]. The models can be further improved by simulating erosion and other natural processes. The terrain generation process isn't addressed in the present work.

The generation of the nodes definition should have in account the natural definitions of the terrain, such as the elevation, coastal areas and waterways. Also, constraints such as a previous road network, edifications and natural parks (forests, cultivation areas). If the preparer of the models doesn't provide a nodes definition, the Nodes module procedurally generates a simplified definition of nodes on a given terrain area. The Nodes module automatically produces a simplified definition of nodes on a given area of the terrain. Each node has a location and an importance factor associated, as illustrated in Fig. 2.

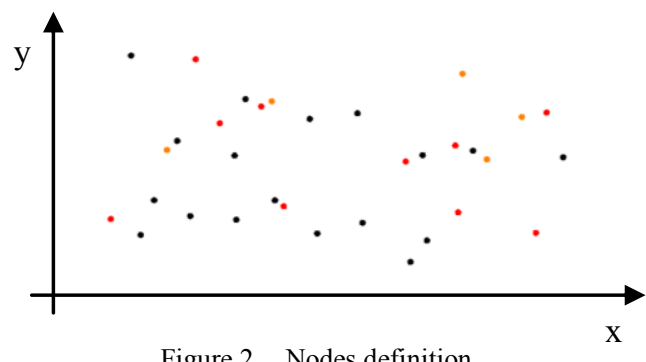

The importance factor may match the population and is used to organize the nodes by typology. The nodes are organized into three typologies: city (black), town (red) and village (orange), as illustrated in Fig. 2. Once the definition of nodes is available, different typological roads networks are created: highways, secondary and rural.

\section{B. Topologic Road Network}

The process starts from nodes of city typology, creating a network of highways. This nodes are organized in a list, by the 
chosen method (population or traffic) and attached to the graph, the distance between all pair of nodes (Fig. 3).

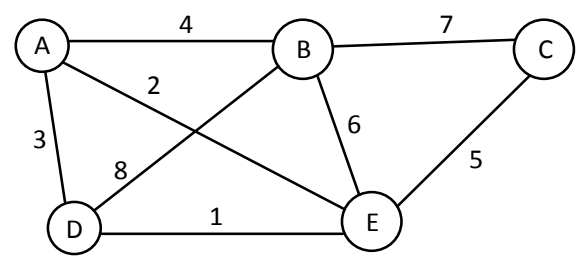

Figure 3. Road network optimization

All considered connections, linking a pair of cities are analyzed. In the network optimization process the benefit and cost of each connection is evaluated. During the review process, the decision of keeping a connection between a pair of nodes $\left(\mathrm{N}_{\mathrm{i}}\right.$ and $\mathrm{N}_{\mathrm{j}}$ ) depends on the difference between the cost of the road construction and the benefit of keeping the connection.

The benefit is related to the utilization index (U), which depends on the resident population in each of the end nodes $\left(\mathrm{P}_{\mathrm{i}}\right.$ and $\mathrm{P}_{\mathrm{j}}$ ) and the total length (L) of the roadway, according to (3).

$$
\mathrm{U}=\mathrm{P}_{\mathrm{i}} * \mathrm{P}_{\mathrm{j}} / \mathrm{L}^{2}
$$

The benefit (B) is determined according to the utilization index $\mathrm{U}$, the length of the connection $\left(\mathrm{L}_{\text {connection }}\right)$ and the shortest alternative route $\left(\mathrm{L}_{\text {shortest }}\right)$, between the nodes $\mathrm{N}_{\mathrm{i}}$ and $\mathrm{N}_{\mathrm{j}}$ (Dijkstra), and the cost of operation $\left(\mathrm{C}_{\mathrm{op}}\right)$, which measures the traveling cost of a vehicle per kilometer (4).

$$
\mathrm{B}=\mathrm{U} *\left(\mathrm{~L}_{\text {shortest }}-\mathrm{L}_{\text {connection }}\right) * \mathrm{C}_{\mathrm{op}}
$$

The connection is removed if the benefit $(\mathrm{B})$ is lower than the construction cost of the road. The connection is maintained if the benefit is greater than the construction cost of the road.

In the optimization process, it is ensured that one node of any type (city, town or village) doesn't end up without any connection to the network. This condition guarantees the existence of at least one road to connect this node to the road network.

After simplifying the road network, there can be intersections between roads. In this case, additional interconnection nodes are generated. These nodes have an associated importance factor (population) of zero. Interconnection nodes with zero population appear as gray nodes in Fig. 5 and 6.

In order to generate the secondary road network, the nodes with village typology are added to the highways network obtained in the optimization process. All possible connections between villages and towns are then analyzed, using the same procedure applied to the highway network. The connections of the previous highway network aren't reanalyzed, analyzing only the new connections in this stage: city-town and towntown.

Lastly, the rural road network is analyzed, connecting village nodes to the previous road network generated. The optimization process is the same that is applied to the previous networks, highway and secondary.
The process of generating the topological road networks may be parameterized in order to obtain a representation of roads (highway, secondary and rural), adequate and similar to a real road network. The parameters that user can set are: Classification index of nodes by type (city, town and village); Construction and operation cost of each road type (highway, secondary and rural); Optimization method of network (population or traffic).

At the end of the process, we obtain a definition of the network composed by the connections between pairs of nodes, as shown in Fig. 6. After obtaining the definition of the topological road network, the generation of the road path for each roadway takes place. Finally the 3D model of the roads and the terrain are created, using procedural techniques previously described in [4].

\section{ROAD NETWORK APLICATION}

In this work an interactive application was also developed in order to allow the testing and evaluation of the proposed method. This application was developed in $\mathrm{C}++$, using Windows Forms to implement interaction elements and the OpenGL library for the main graphical visualization.

The developed application allows to control, in real-time, all the parameters related to the generation of a road network. Simultaneously, the resulting network is displayed in the left window, allowing an interactive customization of the result. As an example, through the application the user can change the threshold used in the classification of the nodes by type and visualize their distribution in the global map. Changing this threshold may result in a redistribution of the typology of nodes and thus, in a redesigned network. The initial definition of nodes comes from a text file that the user can easily edit. The road network generation process starts by loading this file to the application, through the button "Load File" (Fig. 4).
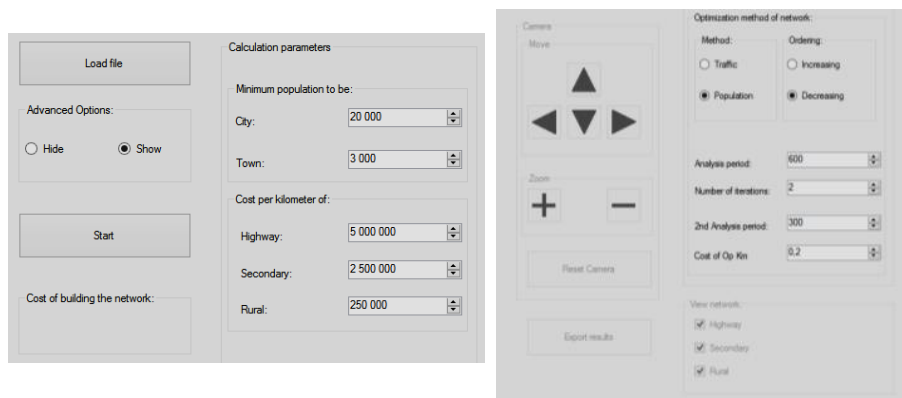

Figure 4. Application control panel

As mentioned in section III.B, the user can edit the parameters of the network generation, such as: classification of nodes typology, construction cost and operation cost, to address the particular requirements of an experimental work.

\section{RESUlTS}

To demonstrate the quality of the results obtained by this method and to provide a critical analysis, a case study was prepared, with nodes that correspond to Portuguese cities, towns and villages. This way, a better analysis can be performed comparing the road network procedural obtained with the real road network, in Portugal. 
In this study case 33 nodes were used, including for example: Braga, Lisboa, Porto, Viana do Castelo, Vila Real, Viseu, Barrancos, Freixo de Espada Cinta and Vidigueira. The population associated to each node was obtained from a Portuguese study called Censos 2011, from the Instituto Nacional de Estatística [19].

To evaluate the proposed method, a real-world terrain definition was used, which was obtained from an online repository [20].

To hierarchize the nodes, the following classification was used:

- City: greater than or equal to 20,000 inhabitants;

- Town: greater than or equal to 3,000 and less than 20,000 inhabitants;

- Village: less than 3,000 inhabitants.

In the first step of the generation process, only the highway network was generated, resulting in the network presented in Fig. 5.

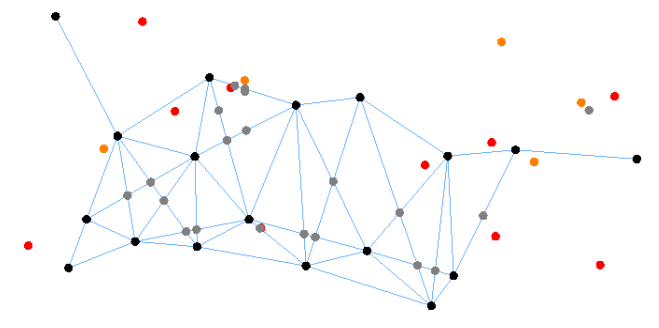

Figure 5. Highways network

As can also be seen in Fig. 5, at this stage, only the nodes of type city (black) are interconnected by highways (blue).

The nodes of type town (red) and village (orange) are still not connected. At this stage, several new interconnection nodes have been created, represented by gray color.

After running the algorithm, the road network showed in the Fig. 6 , is obtained.

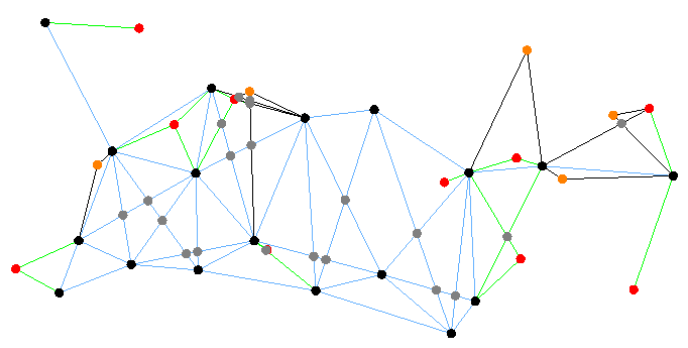

Figure 6. Topological roads network

As can also be seen in the Fig. 6, all nodes (city, town and village) are now connected by a road. The highway network is presented with blue color, the secondary network with green color and villages are connected by rural roads with black color. After obtaining the road network definition, the user can export it to a XML formatted file, as exemplified in Fig. 7.

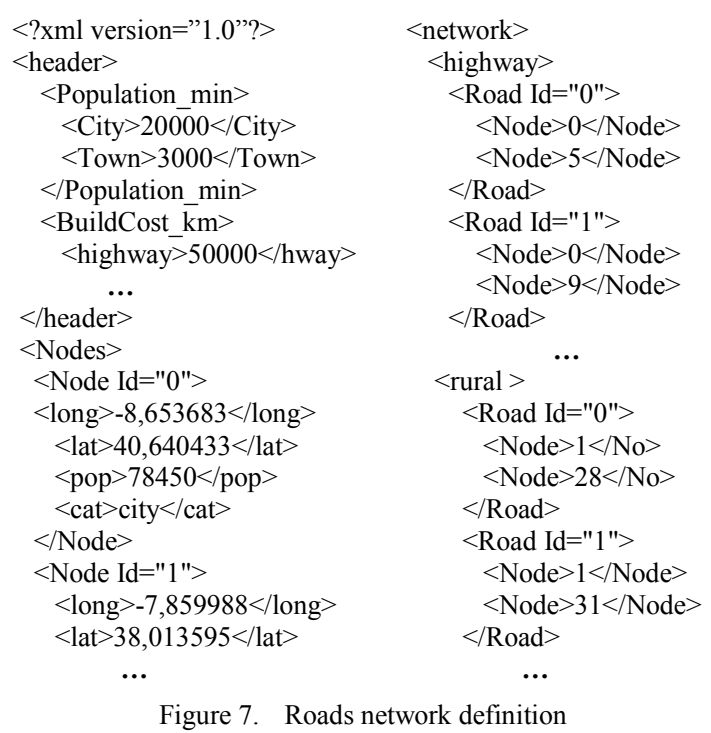

The rendering of the road environment takes place in real time using the simulation system of the driving simulator (Fig. $8)$.

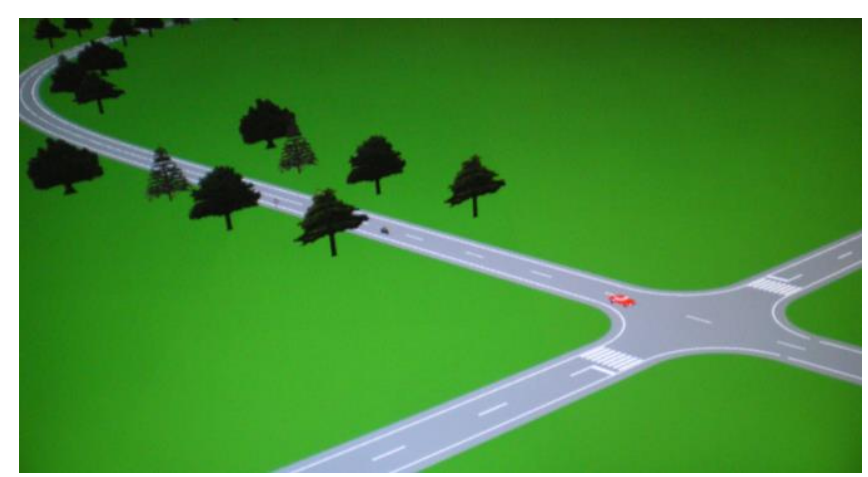

Figure 8. Road network environment.

A special characteristic of our approach is the efficiency and control, to produce a network definition. In table 1, we present the time measured to obtain a topological road definition by our method, with different number of input nodes [1].

TABLE 1. TOTAL NUMBER OF NODES (CITY, TOWN, VILLAGE, AND INTERCONNECTION NODES), TOTAL LENGTH OF THE ROADS (IN KILOMETRES), TIME (IN SECONDS) TO GENERATE DE TOPOLOGICAL ROAD DEFINITION.

\begin{tabular}{|c|c|c|c|c|c|}
\hline $\begin{array}{c}\text { Total } \\
\text { Nodes }\end{array}$ & Cities & Towns & Villages & $\begin{array}{c}\text { Total } \\
\text { Length }\end{array}$ & Time \\
\hline 142 & 25 & 16 & 15 & 7620 & 11 \\
\hline 342 & 25 & 16 & 15 & 12166 & 76 \\
\hline 30 & 18 & 0 & 0 & 3719.2 & 2 \\
\hline
\end{tabular}

Analyzing the highway network in terms of its extension, we conclude that the extension of the real highway network and the generated highway network are approximately the same magnitude length, as show in table 2 [21]. 


\section{ACKNOWLEDGMENT}

TABLE 2. LENGTH OF PROCEDURAL ROAD NETWORK GENERATED AND REAL ROAD NETWORK (IN KILOMETRES).

\begin{tabular}{|c|c|c|}
\hline Type of Network & Real road network & Procedural road network \\
\hline highway & 3770 & 3719.2 \\
\hline
\end{tabular}

Analyzing the results obtained with the procedural method and the real highway roads network in Portugal, we conclude that the designed method for the generation of topological roads network, produces a definition with quality and similar to those found in the real roads network. The secondary and rural road network can't be strictly compared, because the number of town and village nodes used, is less than in the real world.

\section{CONCLUSIONS}

Realistic driving simulation experiences require prior preparation of accurately modeled road environments, with similar characteristics to those found in real roads. Some experiments also require models that are recognizable as roads from the specific simulated country or region.

The presented method allows the procedural generation of a wide variety of topological road network definition, adequate for procedural creation of road environments for driving simulation. The process of generating the road network takes into account the typological classification of the nodes, producing different types of road networks: highway, secondary and rural. The generation of a topological road network is processed at different levels (highway, secondary and rural). In each level the network is analyzed as a whole, producing an optimized result. The optimization is performed by using a cost function that evaluates the estimated construction cost and the benefit of the road. The benefit of a road is calculated based on population of the end nodes. Throughout the process of automatic generation, each typological network is individually evaluated in order to produce an optimized definition of the global road network.

The preparer of the road network can parameterize the optimization process by adjusting the controls parameters, achieving a totally controllable generation process.

This work results in an innovative contribution to the procedural generation of extensive and realistic road networks definition, dramatically reducing the labor and costs involved in the design of the definition of a road network (shown in table 1), and also significantly reducing the involvement of specialists in roads engineering. The main application of this work will be incorporated in a framework to the generation of road environments for driving simulation, aimed to scientific studies in several scientific areas. As shown in results section (Fig. 8), the generated definition of road networks, can be already integrated in the preparation of road environments.

In the near future, the generation of the terrain model will be addressed and the nodes generation module will be improved, producing a distribution that takes into account the features of the terrain, such as the location of coastal areas and watercourses.
The Media Arts and Technologies project (MAT), NORTE07-0124-FEDER-000061, is financed by the North Portugal Regional Operational Program (ON.2 - O Novo Norte), under the National Strategic Reference Framework (NSRF), through the European Regional Development Fund (ERDF), and by national funds, through the Portuguese funding agency, Fundação para a Ciência e a Tecnologia (FCT).

\section{REFERENCES}

[1] Campos, C.; Leitão, J.; Coelho, A., "Integrated Modeling of Road Environments for Driving Simulation", 10 th International Conference on Computer Graphics Theory and Applications, 2015.

[2] Teoh, S., "Algorithms for the Automatic Generation of Urban Streets and Buildings", Proceedings of the International Conference on Computer Graphics and Virtual Reality (CGVR'08), 2008.

[3] Campos, C.; Leitão, J.; Coelho, A., "Geração Procedimental de Traçados Rodoviários para Simulação de Condução", 21. ${ }^{\circ}$ Encontro Português de Computação Gráfica, Novembro de 2014.

[4] Campos, C.; Leitão, J.; Coelho, A., "Building Virtual Roads from Computer made Projects", 17th International Conference on HumanComputer Interaction, in press, 2015.

[5] Weber, B.; Müller, P.; Wonka, P.; Gross, M., "Interactive Geometric Simulation of 4D Cities", Eurographics, vol. 28, 2009.

[6] Parish, Y.; Muller, P., "Procedural Modeling of Cities", ACM Computer Graphics (Proceedings of SIGGRAPH'2001), pp. 301-308, 2001.

[7] Chen,G.; Esch,G.; Wonka,P.; Muller,P.; Zhang,E., "Interactive Procedural Street Modeling", ACM SIGGRAPH, 2008.

[8] Coelho, António; Bessa, Maximino; Sousa, A. Augusto; Ferreira F. Nunes, "Expeditious modeling of virtual urban environments with geo spatial L-systems"; Computer Graphics Forum, Vol. 26, N. 4, pp. 769782, 2007.

[9] Kelly, G.; McCabe, H., "Citygen: An Interactive System for Procedural City Generation", GDTW, UK, 2008.

[10] Sun, J.; Baciu, G.; Yu, X.; Green, M., "Template-Based Generation of Road Networks for Virtual City Modeling”, VRST’02, pp 33-40, NY USA, 2002.

[11] Geuter, S.; Parker, J.; Stewart, N.; Leach, G., "Real-Time Procedural Generation of 'Pseudo Infinite' Cities", ACM GRAPHITE '03, 2003.

[12] Muller, p.; Wonka, p.; Haegler, S; Ulmer, A.; Gool, L.; "Procedural Modeling of Buildings", ACM SIGGRAPH, 2006.

[13] Ostadabbas, S.; Farshbaf, M.; Nourani, M.; Addepalli, "Layered SemiMarkov road generation for driving simulations". In Intelligent Transportation Systems (ITSC), 14th International IEEE Conference on (pp. 365-370), 2011.

[14] Teoh, S., Autopolis: Allowing User Influence in the Automatic Creation of Realistic Cities, Advances in Visual Computing: Proc. of the 3rd International Symposium on Visual Computing (ISVC'07), 2007.

[15] Galin, E.; Peytavie, A; Guérin, E.; Benes, B., "Authoring Hierarchical Road Networks", Pacific Graphics, Volume 30, 2011.

[16] Thomas, G.; Donikian, S., "Modelling Virtual Cities Dedicated to Behavioural Animation", EUROGRAPHICS 2000, vol. 19(3), 2000.

[17] Donikian, S., VUEMS: A Virtual Urban Environment Modeling System, Computer Graphics International, Proceedings. IEEE, p. 84-92, 1997.

[18] Teoh, Soon Tee. "Riverland: an efficient procedural modeling system for creating realistic-looking terrains." Advances in Visual Computing. Springer Berlin Heidelberg, 468-479, 2009.

[19] INE, Instituto Nacional de Estatística, "Censos 2011", available at ine.pt/investigadores/Quadros/Q101.zip, 2011.

[20] USGS, United States Geological Survey, available at earthexplorer.usgs.gov, 2014.

[21] IMT, Instituto da Mobilidade e dos Transportes, "National road network report", 2011, available at http://www.imt-ip.pt. 